\title{
Nucleon-Nucleon Scattering and Effective Field Theory: Including Pions Non-perturbatively
}

\author{
J. Gegelia \\ Department of Physics, The Flinders University of South Australia, \\ Bedford Park, SA 5042, Australia
}

(May 8, 2018)

\begin{abstract}
Next to leading order effective field theory calculations are performed for ${ }^{1} S_{0}$ $N N$ scattering using subtractive renormalization procedure. One pion exchange and contact interaction potentials are iterated using Lippman-Schwinger equation. Satisfactory fit to the Nijmegen data is obtained for the momenta up to $300 \mathrm{MeV}$ in the centre of mass frame. Phase shifts are also compared with the results of KSW approach where pions are included perturbatively.
\end{abstract}

03.65.Nk, 11.10.Gh, 12.39.Fe, 13.75.Cs. 


\section{INTRODUCTION}

There has been much recent interest in applications of the chiral perturbation theory approach to processes involving an arbitrary number of nucleons [3] introduced by Weinberg [1,2].

For processes involving more than one nucleon Weinberg's power counting is applied to the potential rather than to the scattering amplitude. For $n$-nucleon processes the potential is defined as a sum of $n$-nucleon irreducible time-ordered perturbation theory diagrams. The amplitude is obtained by solving Lippmann-Schwinger equation (or Schrödinger equation).

Iteration of the potential via the Lippmann-Schwinger equation leads to divergences. One could try to regularize the potential and include counter-terms, but due to the nonrenormalizability of the theory one would have to include an infinite number of (counter-)terms with more and more derivatives. Hence, one has either to exactly solve the equation and after subtract divergences explicitly, or otherwise draw all relevant diagrams, subtract them and sum up. There are not any equations for subtractively renormalised amplitudes. Fortunately there exist practically more powerful approach, cut-off EFT [4]. Divergences appearing in diagrams can be regulated using (sharp or smooth) cut-off regularization. One can keep cut-off parameter of the order of the mass of lightest integrated particle and fit coupling constants to the experimental data as was done in ref. [5]. Cut-off EFT is equivalent to subtractively renormalised EFT up to the order of considered accuracy [4,6]:7].

Technically more convenient approach based on a new systematic power counting has been suggested by Kaplan, Savage and Wise (KSW counting) in [8]. Pions are included perturbatively within this new scheme. A similar power counting with perturbative pions has been suggested by Lutz [9]. As was mentioned in [10] the expansion parameter in KSW counting is rather large. It raises a question of the usefulness of suggested expansion. Arguments supporting as well as criticising the perturbative inclusion of pions have been given in the literature (see [11] and citations therein. [11] also contains references to lots of papers devoted to EFT study of $N N$ interaction problem).

In Weinberg's power counting the one pion exchange potential is of leading order and hence it has to be iterated via the Lippmann-Schwinger equation. KSW counting suggests that contributions containing multiple iterations of the one-pion exchange potential are higher order and hence need not be included in low order calculations.

In this letter the results of the application of subtractive renormalization procedure to the Weinberg's approach to ${ }^{1} S_{0} N N$ scattering problem are briefly discussed (the details will be given in separate paper). Leading order (contact interaction plus one-pion exchange) plus nextto-leading order contact interaction potential is iterated using Lippman-Schwinger equation.

According Weinberg's power counting the leading order potential for ${ }^{1} S_{0} N N$ scattering is the following:

$$
V_{0}\left(\mathbf{p}, \mathbf{p}^{\prime}\right)=\tilde{C}+V_{\pi}\left(\mathbf{p}, \mathbf{p}^{\prime}\right)
$$

where

$$
\tilde{C} \equiv C+\frac{g_{A}^{2}}{2 f_{\pi}^{2}}, \quad V_{\pi}\left(\mathbf{p}, \mathbf{p}^{\prime}\right) \equiv-\frac{4 \pi \alpha_{\pi}}{\left(\mathbf{q}^{2}+m_{\pi}^{2}\right)}, \quad \alpha_{\pi} \equiv \frac{g_{A}^{2} m_{\pi}^{2}}{8 \pi f_{\pi}^{2}}
$$

$C$ is determined by contact interaction terms in the effective Lagrangian [2], $\mathbf{q}=\mathbf{p}-\mathbf{p}^{\prime}$, 


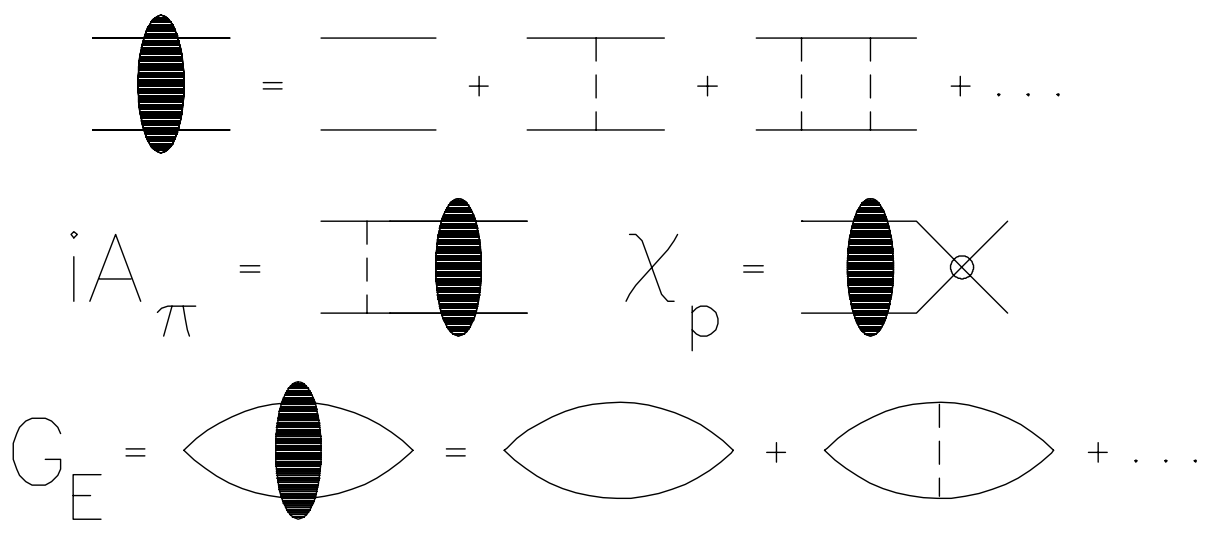

FIG. 1. Diagrams defining $A_{\pi}, \chi_{\mathbf{p}}$ and $G_{E}$. Solid and dashed lines correspond to nucleon and pion respectively. Small circle in the diagram defining $\chi_{\mathbf{p}}$ indicates that factor $\tilde{C}$ is excluded from that vertex.

$g_{A}=1.25$ is the axial coupling constant, $m_{\pi}=140 \mathrm{MeV}$ is the pion mass and $f_{\pi}=132 \mathrm{MeV}$ is the pion decay constant.

Substituting $V_{0}$ into the Lippman-Schwinger equation one finds for the Feynman amplitude [12]:

$$
i A=i A_{\pi}-i \frac{\tilde{C}\left[\chi_{\mathbf{p}}\right]^{2}}{1-\tilde{C} G_{E}}
$$

where the quantities $A_{\pi}, \chi_{\mathbf{p}}$ and $G_{E}$ correspond to diagrams drawn in FIG. $1 . A_{\pi}$ and $\chi_{\mathbf{p}}$ are finite. The first diagram contributing to $G_{E}$ is linearly and the second logarithmically divergent, all other diagrams are finite. As $A$ contains divergences it is necessary to regularize and renormalize it.

Let us introduce a cut-off into the potential:

$$
V_{0}^{\Lambda}\left(\mathbf{p}, \mathbf{p}^{\prime}\right)=\frac{\tilde{C} \Lambda^{8}}{\left(\mathbf{p}^{2}+\Lambda^{2}\right)^{2}\left(\mathbf{p}^{\prime 2}+\Lambda^{2}\right)^{2}}+V_{\pi}\left(\mathbf{p}, \mathbf{p}^{\prime}\right)
$$

where $\Lambda$ is a cut-off parameter.

Substituting $V_{0}^{\Lambda}$ into the Lippmann-Schwinger equation one gets the following expression for the amplitude:

$$
i A^{\Lambda}=i A_{\pi}-i \frac{\tilde{C}\left[\chi_{\mathbf{p}}^{\Lambda}\right]^{2}}{1-\tilde{C} G_{E}^{\Lambda}}
$$

where $A_{\pi}$ is unchanged, $\chi_{\mathbf{p}}^{\Lambda}$ and $G_{E}^{\Lambda}$ are given by the same diagrams in FIG.1 with $\tilde{C}$ replaced by $\frac{\tilde{C} \Lambda^{8}}{\left(\mathbf{p}^{2}+\Lambda^{2}\right)^{2}\left(\mathbf{p}^{\prime 2}+\Lambda^{2}\right)^{2}}$.

Note that $\chi_{\mathbf{p}}^{\Lambda}=\chi_{\mathbf{p}}+0(1 / \Lambda)$ and $G_{E}^{\Lambda}=a_{1} \Lambda+a_{2} \ln \Lambda+G_{E}^{f}+0(1 / \Lambda)$, where $G_{E}^{f}$ is finite and $\Lambda$-independent. It is straightforward to find $a_{1}$ and $a_{2}$ by calculating analytically first two diagrams contributing to $G_{E}^{\Lambda}$. 


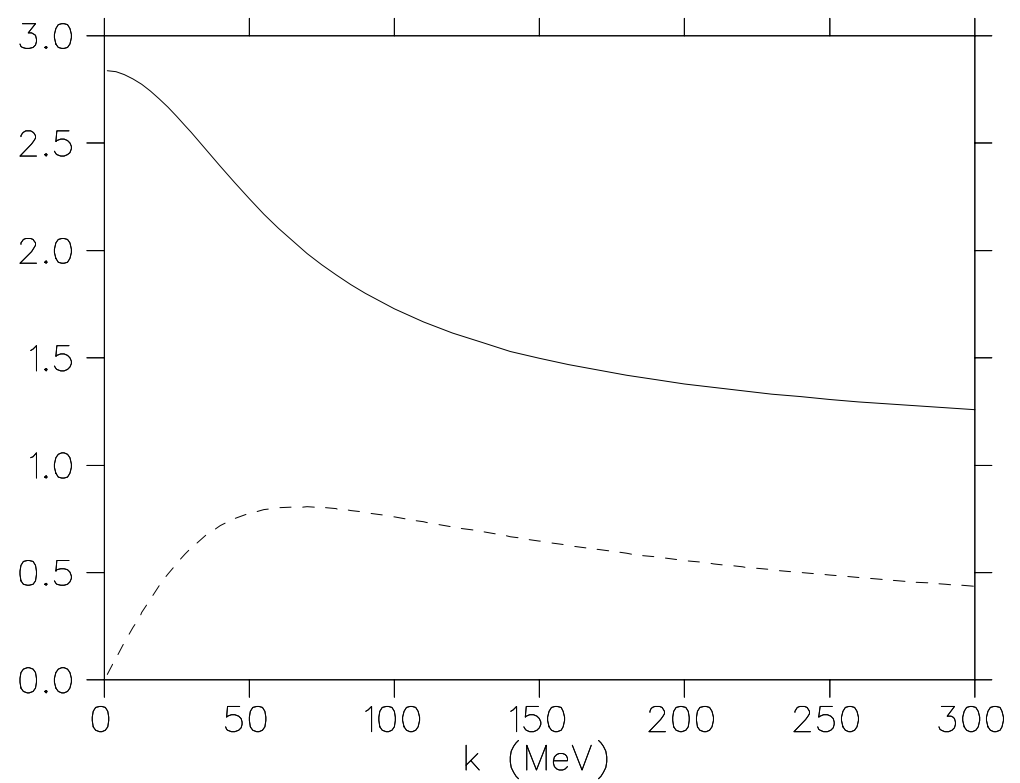

FIG. 2. $\left[\chi_{\mathbf{p}}(\mathbf{0})\right]^{2}$ plotted versus momentum in $\mathrm{MeV}$. The solid and dashed lines correspond to the real and imaginary parts respectively.

One can numerically calculate $A_{\pi}$ and $A^{\Lambda}$ for different values of $\Lambda$. Using these values of $A_{\pi}$ and $A^{\Lambda}$ one finds:

$$
\frac{1}{A^{\Lambda}-A_{\pi}}=B_{1}(p) \Lambda+B_{2}(p) \ln \Lambda+B_{3}(p)
$$

Although $B_{3}(p)$ actually depends on $\Lambda$, for very large $\Lambda$ it practically does not and $B_{1}(p)$ and $B_{2}(p)$ can be calculated very accurately. Having calculated $a_{1}, a_{2}, B_{1}(p)$ and $B_{2}(p)$ one easily finds:

$$
\begin{gathered}
{\left[\chi_{\mathbf{p}}^{\Lambda}\right]^{2}=\frac{a_{1}}{B_{1}(p)}} \\
{\left[\chi_{\mathbf{p}}^{\Lambda}\right]^{2}=\frac{a_{2}}{B_{2}(p)}}
\end{gathered}
$$

Good agreement between numerical results obtained from (7) and (\$) suggests that numerical calculations are enough accurate. $\left[\chi_{\mathbf{p}}\right]^{2}$ obtained this way is plotted in FIG. 2 . The same quantity was calculated in [12] using a different method. The agreement between FIG. 2 and the results of [12] is quite satisfactory.

Having calculated $\left[\chi_{\mathbf{p}}^{\Lambda}\right]^{2}\left(\approx\left[\chi_{\mathbf{p}}\right]^{2}\right)$ it is not difficult to calculate the sum $\tilde{G}_{E}^{\Lambda}\left(\approx \tilde{G}_{E}\right)$ of all finite regularised (unregularised) diagrams contributing to $G_{E}^{\Lambda}\left(G_{E}\right)$.

Phase shifts corresponding to subtractive renormalization procedure can be calculated performing following steps:

1. Calculate $A_{\pi}$, calculate $A^{\Lambda}$ for very large $\Lambda\left(\Lambda \sim 10^{5} \mathrm{MeV}\right.$ has been taken $)$;

2. Calculate 


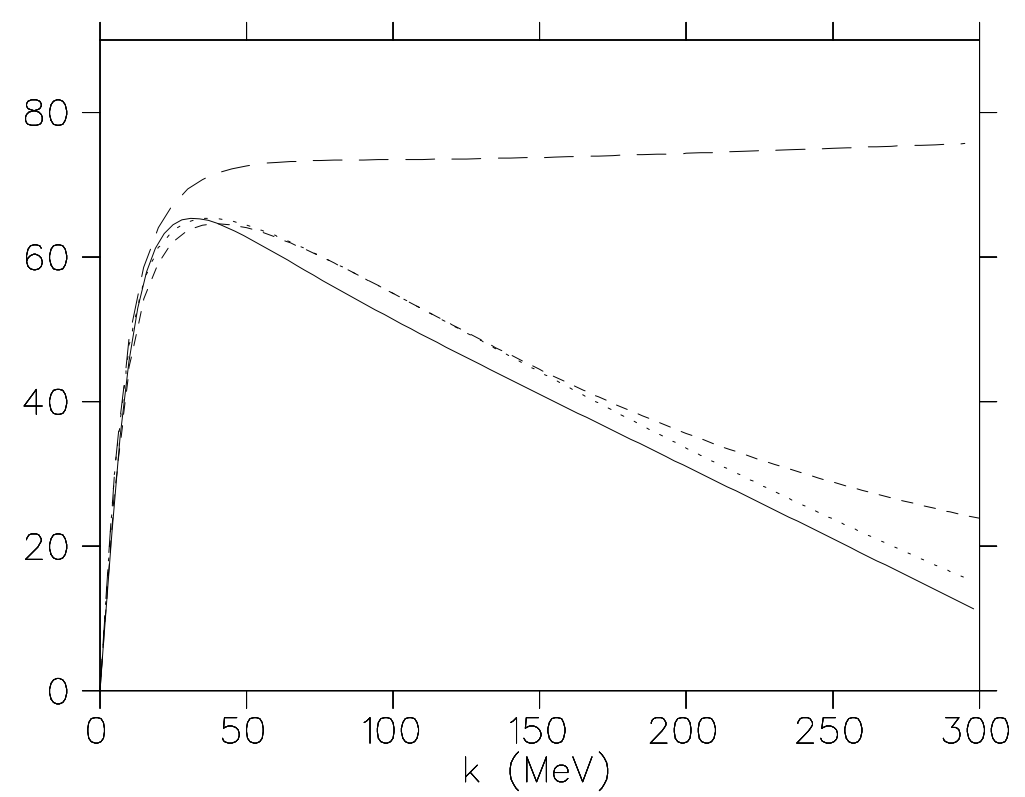

FIG. 3. Phase shifts in degrees versus centre of mass momentum. Solid line corresponds to Nijmegen phase shift data. Long-dashed line is the leading order EFT result. Dotted and short-dashed lines are the results of next-to-leading order calculations. The dotted and small-dashed lines correspond to non-perturbative and non-perturbative inclusion of next-to-leading order potential $C_{2}\left(\mathbf{p}^{2}+\mathbf{p}^{\prime 2}\right) / 2$.

$$
W(p)=-\frac{1}{\tilde{C}}+\tilde{G}_{E}^{\Lambda}=\frac{\left[\chi_{\mathbf{p}}^{\Lambda}\right]^{2}}{A^{\Lambda}-A_{\pi}}-I^{\Lambda}(p)-I_{\pi}^{\Lambda}(p)
$$

where $I^{\Lambda}(p)$ and $I_{\pi}^{\Lambda}(p)$ correspond to the first and second divergent diagrams contributing to $G_{E}^{\Lambda}$ respectively.

3. Calculate two divergent diagrams contributing to $G_{E}$ analytically, subtract the second logarithmically divergent diagram at $p^{2}=-\mu^{2}$ and the first linearly divergent diagram at $p^{2}=$ $-\nu^{2}\left(=-\kappa \mu^{2}\right)$ (one is free to subtract these two diagrams at different points). In calculations of this work $\mu=240 \mathrm{MeV}$ and $\nu=800 \mathrm{MeV}$ were taken $(\kappa=100 / 9)$. Note that the large value of the second subtraction point $(800 \mathrm{MeV})$ does not affect the power counting.

Subtracted diagrams give:

$$
I_{\pi}^{R}(p)=\frac{g_{A}^{2}}{2 f^{2}}\left(\frac{m_{\pi} M}{4 \pi}\right)^{2}\left[i \tan ^{-1}\left(\frac{2 p}{m_{\pi}}\right)-\frac{1}{2} \ln \left(1+\frac{4 p^{2}}{m_{\pi}^{2}}\right)+\ln \left(1+\frac{2 \mu}{m_{\pi}}\right)\right]
$$

and

$$
I^{R}(p)=\frac{M(\nu+i p)}{4 \pi}
$$

4. Calculate renormalized amplitude:

$$
A^{R}=A_{\pi}+\frac{\left[\chi_{\mathbf{p}}^{\Lambda}\right]^{2}}{W(p)+I^{R}(p)+I_{\pi}^{R}(p)}
$$


5. Fit $C$ so as to reproduce phase shifts for very low energies $(p \sim 5 \mathrm{MeV})$. One gets $C=-1 /\left(17348 \mathrm{MeV}^{2}\right)$.

6. Calculate phase shifts. Comparing phase shifts calculated for $\Lambda=1 \times 10^{5} \mathrm{MeV}, 2 \times$ $10^{5} \mathrm{MeV}, 3 \times 10^{5} \mathrm{MeV}, 4 \times 10^{5} \mathrm{MeV}$ one sees that $A^{R}$ is practically cut-off independent for very large $\Lambda$.

These leading order phase shifts are plotted in FIG. 3.

As was argued in [12] $C_{2}$ coupling constant of the part of the next-to-leading order potential

$$
V_{1}=C_{2}\left(\mathbf{p}^{2}+\mathbf{p}^{\prime 2}\right) / 2
$$

is larger than assumed by Weinberg's power counting. This suggests that contributions of this part of the next-to-leading order potential are larger than of others. This observation simplifies the actual next-to-leading order calculations significantly. It is possible to sum up all diagrams obtained by iterating Lippman-Schwinger equation for the potential $V=V_{0}+V_{1}$, where $V_{0}$ and $V_{1}$ are given by (1) and (13) respectively. The result obtained in [12] using dimensional regularization reads:

$$
A_{1}(\mathbf{p})=A_{\pi}-\frac{\left[\chi_{\mathbf{p}}\right]^{2}}{\left[\tilde{C}-\alpha_{\pi} m_{\pi} M C_{2}+C_{2} \mathbf{p}^{2}\right]^{-1}-G_{E}}
$$

As $\chi_{\mathbf{p}}$ and $\tilde{G}_{E}$ are already calculated (although these quantities were calculated using cut-off regularization, as was mentioned above the effect of finite cut-off is extremally small and hence negligible), it is straightforward to apply the same subtraction scheme to (14), fix $C_{2}$ so as to fit the data for $p=10 \mathrm{MeV}$ and calculate the phase shifts. The results are plotted in FIG. 3. One can see that agreement with Nijmegen phase shift data 13] is quite good. Obtained value $C_{2}=1 /\left(8.5 \times 10^{9} \mathrm{MeV}^{4}\right)$ is indeed larger than assumed by Weinberg's counting.

One also can include $V_{1}$ perturbatively expanding (14) in $C_{2}$ and retaining only first two terms. Calculated phase shifts are also plotted in FIG. 3. Comparing the phase shifts for perturbative and non-perturbative inclusion of $V_{1}$ one sees that deviation is small for momenta up to $300 \mathrm{MeV}$. This suggests that higher order contributions of $V_{1}$ are small as is expected from power counting arguments.

On the other hand it is straightforward to include pions perturbatively (as was done in [14]) following ideas of KSW counting. Using Those values for $C$ and $C_{2}$ which were obtained above one gets negative phase shifts. this suggests that the higher order contributions of the one pion exchange potential are by no means small. Note that subtraction scheme used here is different from the one considered in [14]. In [14] finite diagrams were subtracted as well. Repeating the calculations of this work with non-perturbatively included one pion exchange potential using PDS scheme and substituting the "best fit" parameters from [8] one gets again negative phase shifts. This comparison of the phase shifts calculated using perturbative and non-perturbative inclusion of pions supports the conclusion of [14] that the consistency of the perturbative inclusion of pions in EFT approach to the $N N$-scattering problem is questionable.

\section{ACKNOWLEDGEMENTS}

This work was carried out whilst the author was a recipient of an Overseas Postgraduate Research Scholarship and a Flinders University Research Scholarship at the Flinders University of South Australia. 


\section{REFERENCES}

[1] S.Weinberg, Phys.Lett. B251 (1990) 288;

[2] S.Weinberg, Nucl.Phys. B363 (1991) 3;

[3] Nuclear Physics with Effective Field Theory, ed. R. Seki, U. van Kolck, and M. J. Savage (World Scientific, 1998);

U. van Kolck, nucl-th/9902015, and references therein.

[4] G.P.Lepage, nucl-th/9706029;

[5] C.Ordonez, L.Ray and U. van Kolck, Phys.Rev. C 53 (1996) 2086;

[6] G.P.Lepage, What is Renormalization?, in From actions to Answers, edited by T.DeGrand and D.Toussaint, World Scientific Press (Singapore,1990).

[7] J.Gegelia, nucl-th/9805008, to be published in Journ. of Phys. G;

[8] D.B.Kaplan, M.J.Savage and M.B.Wise, Phys.Lett. B424 (1998)390, nucl-th/9801034;

[9] M.Lutz, in proceedings of the workshop "The Standard Model at Low Energies" held at ECT* in Trento, Italy, from April 29 to May 10, 1996, hep-ph/9606301; Nucl. Phys. A642 (1998) $171 ;$ nucl-th/9906028;

[10] D.B.Kaplan, M.J.Savage and M.B.Wise, nucl-th/9802075;

[11] D.B.Kaplan, J.V.Steele, nucl-th/9905027;

[12] D.B.Kaplan, M.J.Savage and M.B.Wise, Nucl.Phys. B478 (1996) 629;

[13] Partial wave analysis of the Nijmegen University theoretical high energy physics group, obtained from WorldWide Web page lttp://nn-online.sci.kun.nl/

[14] J. Gegelia, nucl-th/9806028; 